\title{
Exploration and Navigation in Corridor Environment Based on Omni-View Sequence
}

\author{
Yoshio Matsumoto ${ }^{\dagger}$, Kazunori Ikeda $^{\ddagger}$, Masayuki Inaba $^{\ddagger}$ and Hirochika Inoue H $^{\ddagger}$ \\ ${ }^{\dagger}$ Graduate School of Information Science, Nara Institute of Science and Technology \\ 8916-5, Takayamacho, Ikoma-city, Nara, 630-0101, Japan \\ Email: yoshio@is.aist-nara.ac.jp \\ URL: http://robotics.aist-nara.ac.jp/ yoshio \\ ${ }^{\ddagger}$ Graduate School of Engineering, The University of Tokyo
}

\begin{abstract}
A map is a description which represents the structure of an environment. In order for a robot to move from a point to another efficiently in an environment, a map of the environment is the essential information. "Omni-View Sequence" is a kind of route representation which we have developed for robot navigation in a corridor environment. It contains a sequence of omnidirectional views as the information of a route. However it cannot be regarded as a map, because the start and the goal points of the route are both fixed.

In this paper, we first propose a map named "ViewSequenced Map" which can represents a whole corridor environment on a floor in a building. Then we describe the method for acquiring a View-Sequenced Map automatically based on the exploration of a robot in a corridor using both stereo and omnidirectional vision. Finally experimental results of the map acquisition and the autonomous navigation are presented to show the feasibility of the proposed map representation.
\end{abstract}

\section{Introduction}

A map is a description which represents the structure of an environment. In order for a robot to navigate from a point to another in an environment, a map of the environment is the essential information. However in vision-based mobile robotics researches, not many "maps" developed so far have fully visual descriptions in order for a robot to recognize its position in an environment. For instance, the map representation used for Polly[1] contains only the visual information of several discrete places in the corridor. This means the robot can recognize where it is at only some particular places. Panoramic View[2] proposed by Zheng et al. is a route representation based on the memorization of the side view seen along a route. Our naviga- tion method using "View Sequence" [3][4] also utilizes memorized view along a route. Since the start and the goal points are both fixed in such route representations and they don't support a navigation between arbitrary places, neither of them can be regarded as a map. However "T-Net" [5] proposed by Ishiguro et al. is a complete visually described map representation based on Panoramic View. It is generated by merging multiple Panoramic Views through autonomous exploration of a robot.

In this paper, "View Sequence" is extended to a map representation based on the similar idea. We first propose a map called "View-Sequenced Map" which represents a whole corridor environment. Then we describe the method for acquiring a View-Sequenced Map automatically based on the exploration of a robot in a corridor using both stereo and omnidirectional vision. Finally experimental results of the map acquisition and the autonomous navigation are presented to show the feasibility of the proposed method.

\section{View-Sequenced Map}

\subsection{Omni-View Sequence}

"View Sequence" [3] is a route representation to guide a robot along a route. The robot memorizes a sequence of views in front of it while being controlled by a human operator along a route. Although the operation of teaching the route is quite instinctive and easy, it has some limitations. An extended route representation named "Omni-View Sequence" [4] which consists of a sequence of omnidirectional images was proposed to overcome the limitations.

Firstly, the Omni-View Sequence enables the robot to came back using the same data as shown in Figure 1 (a), while the original View Sequence represented only a route from a point to another. This can be 


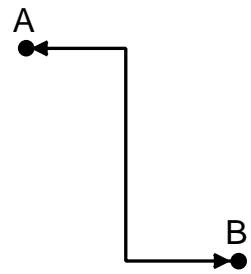

(a)

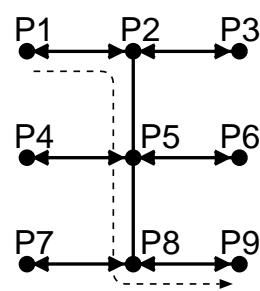

(b)
Fig. 1 : Omni-View Sequence (a) and View Sequenced Map (b).

done by just reversing the order of the views in the sequence and rotating each Omni-View by 180 [deg].

Secondly, since the Omni-View has much wider field of view than a normal camera, the robustness in localization is drastically improved especially at places close to the wall. In such situations, a normal view contains almost only the image of the wall, and the appearance doesn't change sufficiently to memorize another view in the teaching run. In contrast with this, an Omni-View indicates larger change as the robot moves which improves the robustness in the localization process in the autonomous run.

\subsection{Extension to View-Sequenced Map}

As shown in Figure 1 (b), the View-Sequenced Map is an extension of the Omni-View Sequence. However they have different representation forms in some sense. The conventional Omni-View Sequence has so-called a "robot-centered representation" of the route. That is, the center of the views always correspond to the frontal direction of the robot and an action tag attached to each view corresponds to the action which the robot should take when the view is seen.

In contrast, the View-Sequenced Map has a "world representation." The center of each view corresponds to the same direction (e.g. north), and the positional relationship to the neighboring views is attached to each view. The number of neighboring view can be more than two, which makes it possible to represent junctions in the map.

Once a View-Sequenced Map is acquired, it can generate an Omni-View Sequence when given arbitrary start and goal points in the map. For instance, when P1 and P9 are specified as the start and the goal in the map shown in Figure $\mathbf{1}$ (b), an Omni-View Sequence shown in Figure $\mathbf{1}$ (a) is generated. Then a robot can navigate from P1 to P9 using the generated Omni-View Sequence.

\section{Exploration and Acquisition of View-Sequenced Map}

The former way of manually building Omni-View Sequences can be applied for building a View-Sequenced Map. However it is not an easy task for a human operator, since it includes tasks as follows:

- To acquire each Omni-View Sequence in each straight course without the human operator being captured in the image,

- To correct the direction of views in the acquired Omni-View Sequences depending on the direction of the courses,

- To merge multiple Omni-View Sequences by connecting them at proper views which correspond to junctions.

Instead of this manual approach, an approach that the robot explore the corridor environment and build the map by itself is taken. In order to explore in an unknown corridor environment composed of straight courses and junctions, following two functions are necessary for a mobile robot:

\section{Free Space Detection}

\section{Junction Detection}

The robot first starts to move in a straight course to the endpoint based on Free Space Detection, while it memorizes an Omni-View Sequence automatically. During this movement, it also keeps performing Junction Detection by checking free spaces on both sides of the robot. When it finds a junction, it stores the ID number of the corresponding memorized Omni-View in the stack memory. After reaching the endpoint, it takes the ID out of the stack and goes back to the junction using the memorized Omni-View Sequence. Then it starts an exploration in another straight course until it reaches its endpoint. When the stack becomes empty, it is the end of the exploration in the environment. In our research, both of the functions are implemented using vision processing, although they can be easily achieved using range sensors such as ultra sonic sensors.

\subsection{Free Space Detection}

In order for a robot to move to the end of a straight corridor, the robot have to have the capability of detecting the free space in front of it. This function was implemented using our compact stereo vision system $[6]$. Figure 2 illustrates an experimental result of the free space detection. Each circle in the upper image in Figure 2 corresponds to the stereo disparity in the 

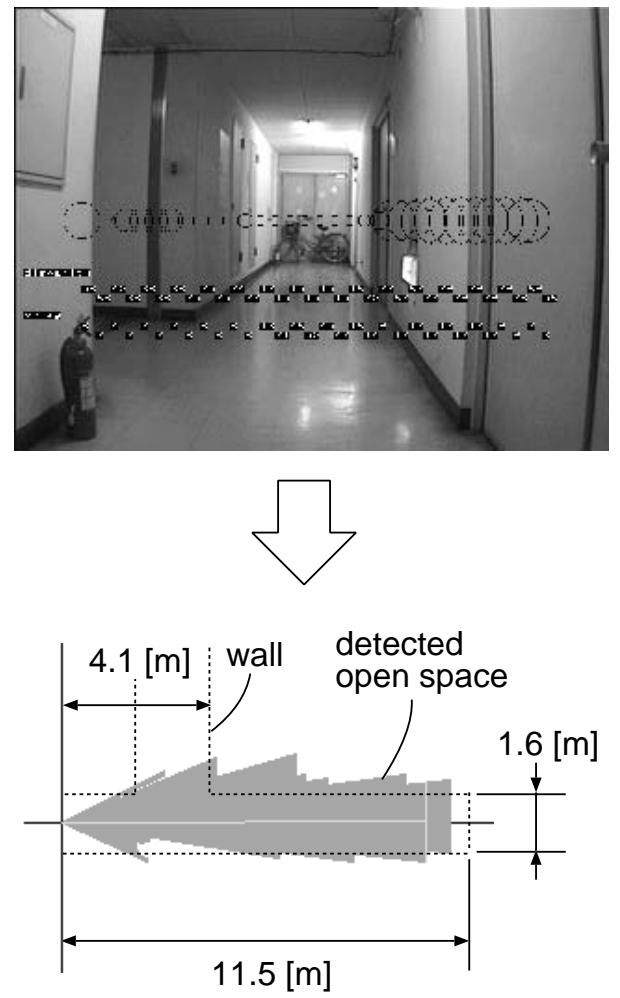

Fig. 2 : Upper: result of stereo matching, lower: detected free space in front of the robot.

stereo image, thus the larger circle indicates the shorter distance from the robot to the object. The disparity information is calculated based on template matching, which takes $137[\mathrm{~ms}]$ for 32 points by the correlation LSI on our vision processing system[7]. The lower figure in Figure $\mathbf{2}$ shows the reconstructed free space in top-view projection. The dotted line indicates the ground truth of the shape of the corridor. The reconstructed result is not very accurate in terms of the geometric shape, but sufficient for a mobile robot to perform the exploration without colliding against the wall. The robot keeps moving to the direction of the furthest point in the free space, while memorizing an Omni-View Sequence.

\subsection{Junction Detection}

Junctions are detected based on the optical flows on the omnidirectional image. The optical flows are assumed to be caused by only the motion of the robot which is going forward. Then the length of the optical flows in the left and the right part of the image should be proportional to the inverse of the distance to the object. Therefore a closer object makes a larger optical flow. This is the basic idea for detecting open spaces on the side of the robot. Figure 3 illustrates

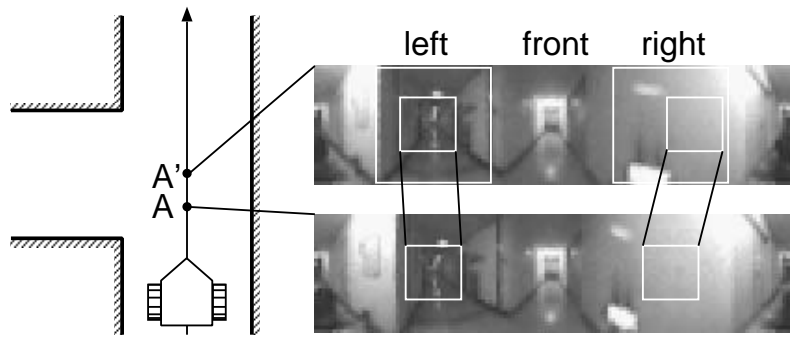

Fig. 3 : Optical flows obtained on both sides of the robot at a junction.
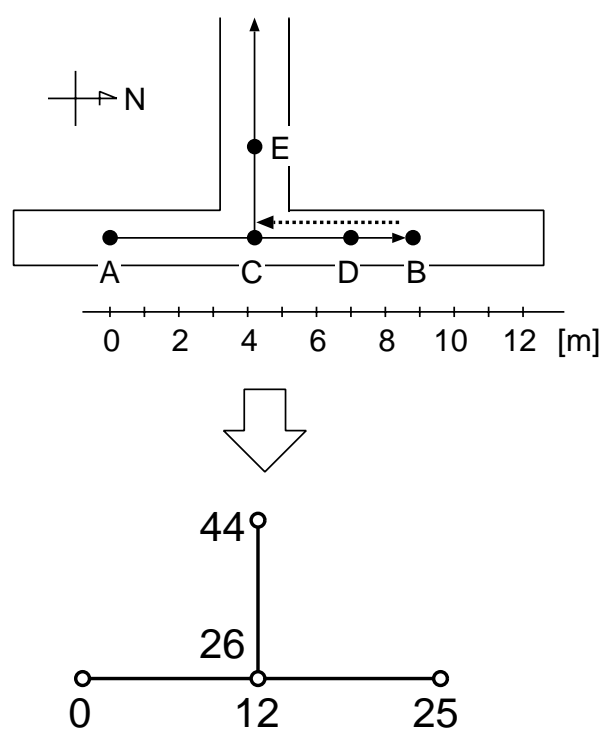

Fig. 4 : Upper: experimental environment, lower: topological structure of acquired map.

an experimental result of optical flow detection at a T-shaped junction. Since there was an open space on the left, the optical flow in the left part of the image became significantly smaller than that on the right.

\subsection{Experimental Results}

The map acquisition should be carried out in a static environment without temporal objects in it. Figure 4 shows the experimental environment. The robot was first placed at the point A, directing to the north. Then the robot started moving toward the point $\mathrm{B}$ based on the free space detection while continuously memorizing the Omni-View Sequence along the route. At point $\mathrm{C}$, it detected a junction on the left and stored the ID information in the stack. It kept going forward using free space detection until it found the end of the corridor at point B. Then it took the ID of the detected junction out of the stack, and went back to the point $\mathrm{C}$ using previously memo- 


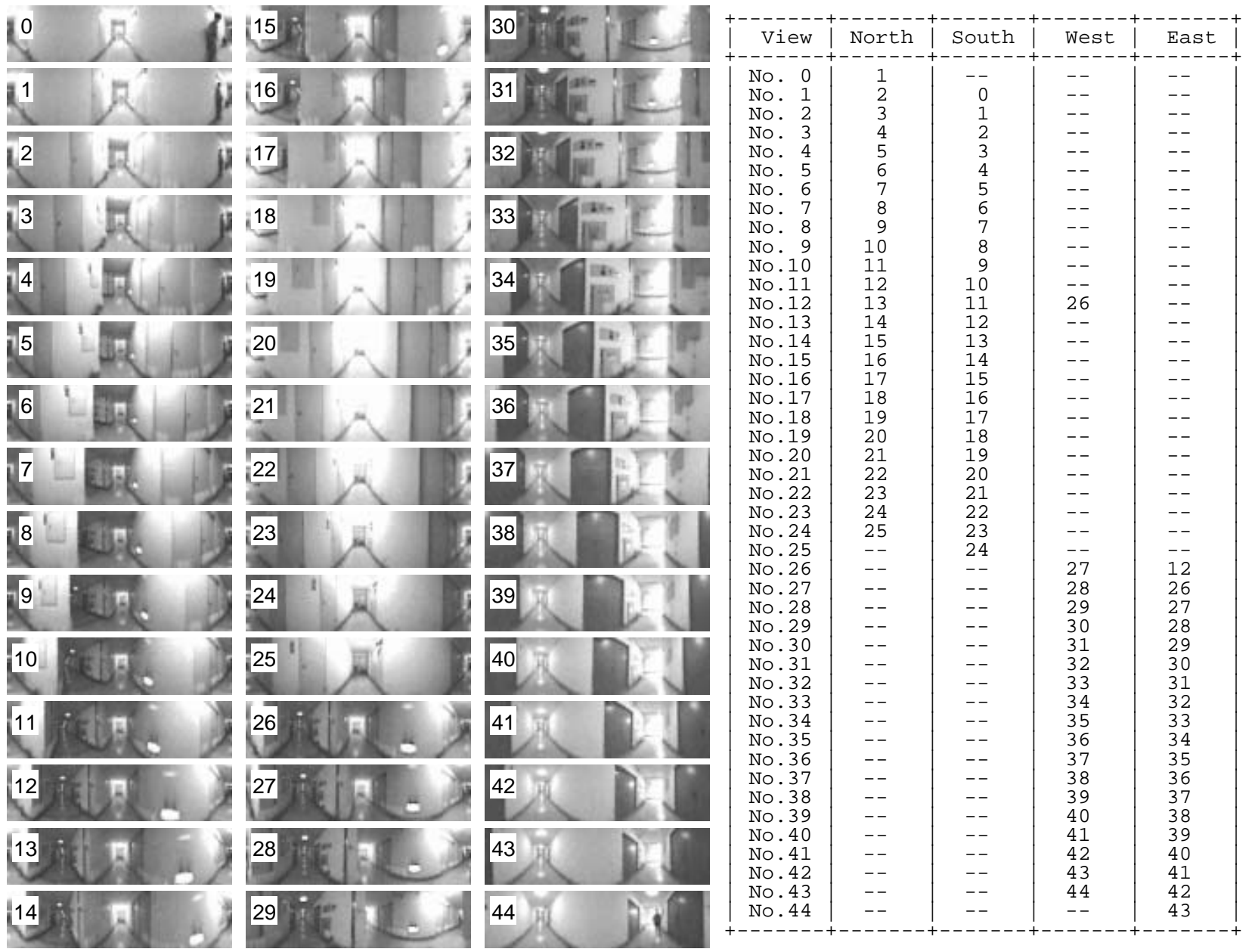

Fig. 5 : Acquired View-Sequenced Map in the experiment.

rized Omni-View Sequence. At C, the robot turned to the west and started exploring another corridor, while memorizing the Omni-Views. The lower figure in Figure 4 indicates the topological shape of the acquired View-Sequenced Map, and Figure 5 shows the acquired View-Sequenced Map.

Figure 6 shows the information displayed on the monitor during the exploration. Detected free space in front and on both sides, together with the current and the last memorized Omni-Views are shown.

After the map of an environment is acquired, the human operator can give names to particular places, such as "Inoue-Lab", "Business Office" and "Elevator". The interface for this operation is shown in Figure 7 . The operator can "walk through" the virtual corridor environment in the acquired map to reach such places.

\section{Autonomous Navigation Based on View-Sequenced Map}

\subsection{Localization}

Before starting the navigation, the robot need to know where it locates in the map. The localization is achieved by the matching of current Omni-View with all Omni-Views in the acquired map. In this experiment, the robot was located at the point D in Figure 4 . The result of global search is shown in Figure 8 . The view No.21 had the minimum matching error, and was regarded as the current position of the robot in the map. The best matched view and its neighboring views are shown in Figure 9 .

\subsection{Generation of Omni-View Sequence}

Then the robot was ordered to go to the point E. This goal was selected randomly by a human operator. This 


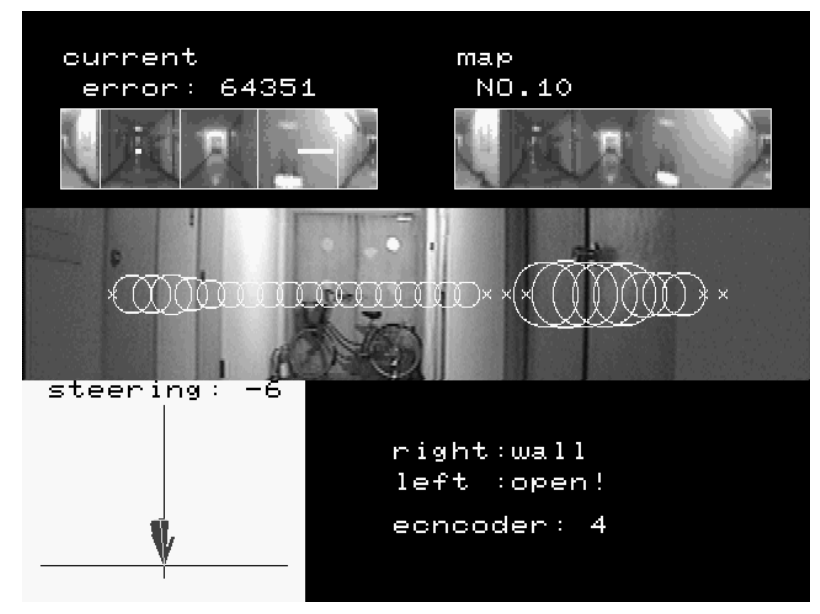

Fig. 6 : Information displayed during exploration.

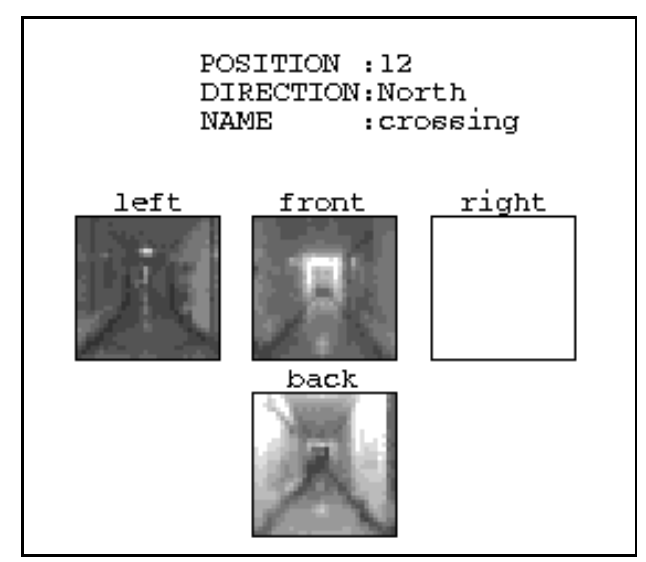

Fig. 7 : Interface for Giving Names for Particular Positions.

can be done by giving the name of the position using either the keyboard or speech recognition. After given the goal of the navigation, the robot plans the shortest path from the current position to the goal, and generates a Omni-View Sequence for the path. This procedure includes the rotation of each Omni-View and the conversion of the positional relationship in the map into the sequence of action to build a "robot-centered representation" as shown in Figure 10. Figure 11 is the generated Omni-View Sequence. Each view is stored together with the corresponding action (e.g. "Go Forward," "Turn Right") which should be taken when the view is seen.

\subsection{Run}

After an Omni-View Sequence to get to the goal is generated, the robot can finally start the navigation. Figure 12 shows the information displayed on the monitor during the navigation.

Current Omni-View and the corresponding Omni-

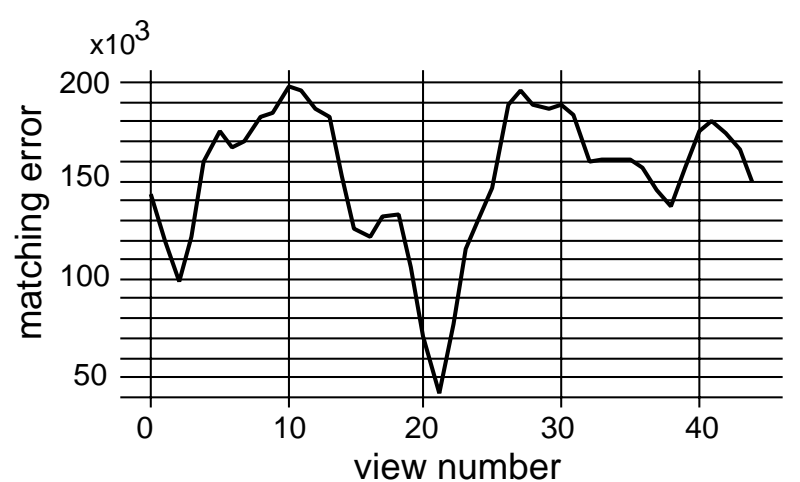

Fig. 8 : Experimental result of localization.

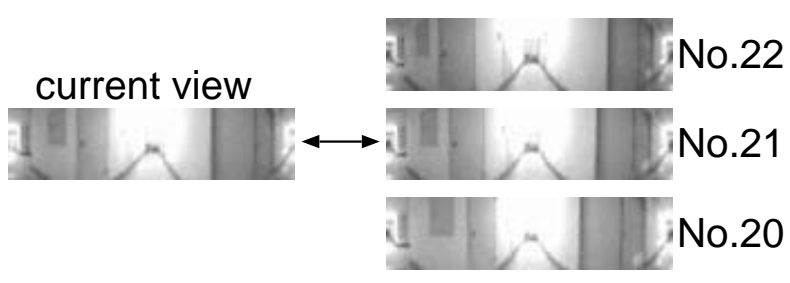

Fig. 9 : Current view and estimated view.

View in the map are shown in the top, while the steering angle and the result of obstacle detection (i.e difference image between the current view and the view in the map) are shown in the bottom in Figure 12 . In the experiment, a person stood in front of the robot as an obstacle, and the figure can be seen as a white region in the difference image. When obstacle is found in the center of the image, the robot stops until it disappears. Then after the person disappeared, the robot successfully arrived at the goal.

\section{Conclusion}

In this paper, the Omni-View Sequence was extended to the View-Sequenced Map, which has a "world representation" of an environment. Then the method of autonomous acquisition of the View-Sequenced Map in a corridor environment was developed using both stereo and omnidirectional vision. Finally experimental results of the map acquisition and the autonomous navigation were presented to show the feasibility of the proposed map representation.

Exactly speaking, the frequency of the memorization varies depending on the complexity of the appearance of the environment. However the Omni-Views were regularly memorized about every $1[\mathrm{~m}]$ in our experiment. Since the size of each Omni-View is $4[\mathrm{kB}]$, the data size of the acquired map containing 45 OmniViews is about $180[\mathrm{kB}]$ which is sufficiently small for a map representation in a building. After the map ac- 


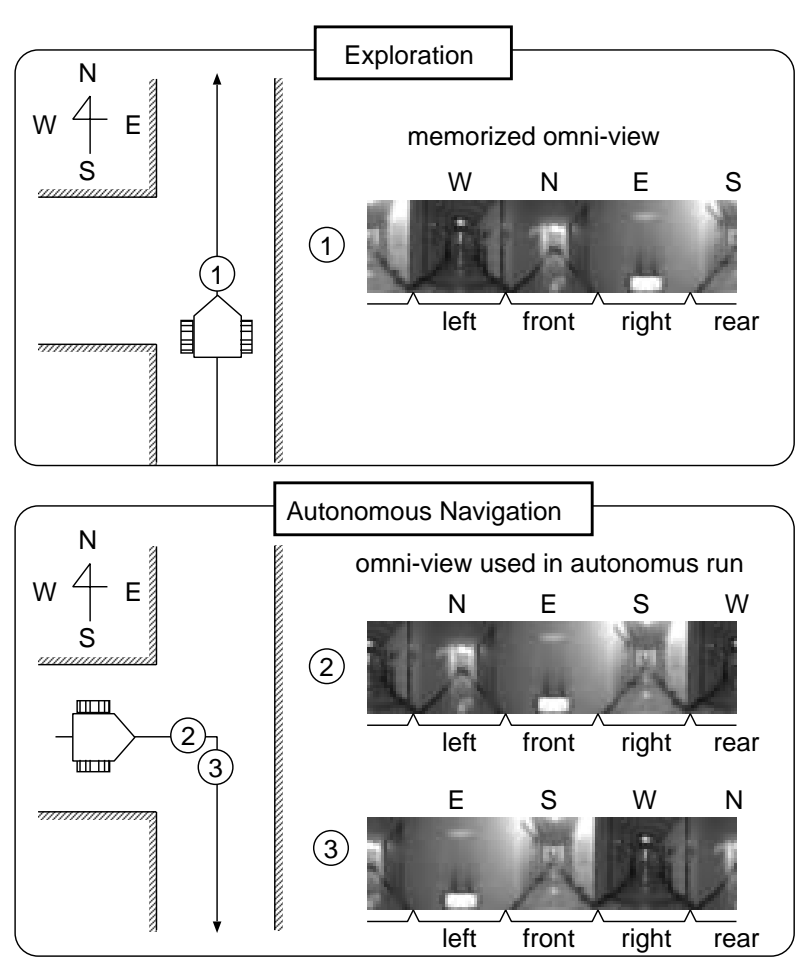

Fig. 10 : Conversion from Map to View Sequence.
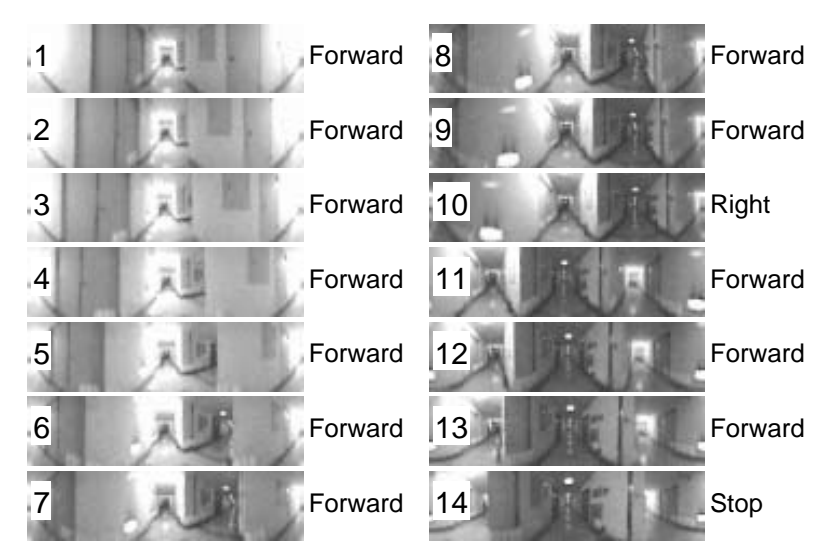

Fig. 11 : Generated Omni-View Sequence.

quisition procedure, the robot can go to an arbitrary place specified by an operator. The application area of such a robot system would be the service robotics such as a "delivery robot" and a "patrol robot" system.

Future works include (1) an acceleration of the map acquisition procedure by utilizing faster stereo matching algorithms, (2) an improvement of the acquisition algorithms to cope with closed loops in an environment and (3) an extension of the map representation for dynamic environments.

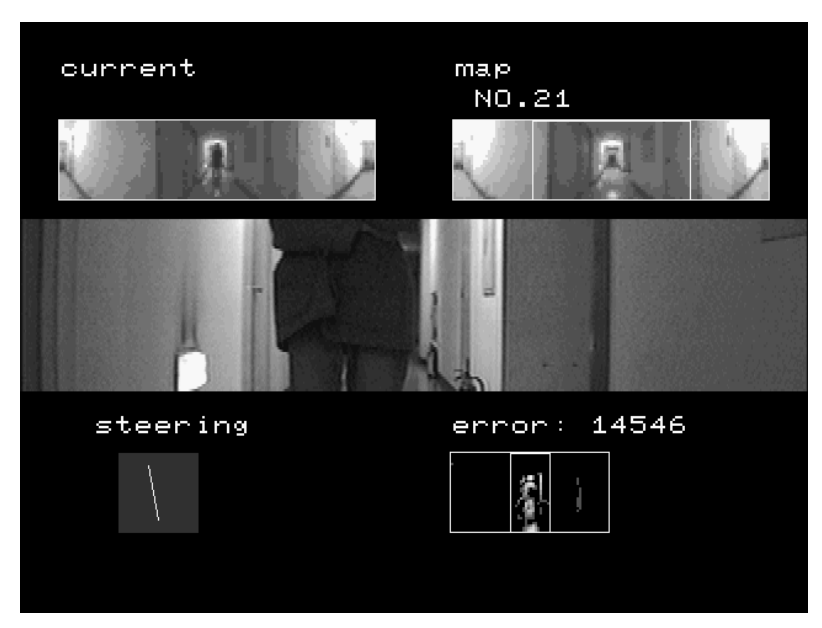

Fig. 12 : Information displayed during autonomous navigation.

\section{References}

[1] I. Horswill. Polly: A Vision-Based Artificial Agent. In Proc. of Int'l Conf. on AAAI '93, pp. 824-829, 1993.

[2] J.Y.Zheng and S.Tsuji. Panoramic Representation for Route Recognition by Mobile Robot. International Journal of Computer Vision, Vol. 9, No. 1, pp. 55-76, 1992.

[3] Y. Matsumoto, M. Inaba, and H. Inoue. Visual Navigation using View-Sequenced Route Representation. In Proc. of IEEE Int. Conf. on Robotics and Automation, pp. 83-88, 1996.

[4] Yoshio Matsutmoto, Kazunori Ikeda, Masayuki Inaba, and Hirochika Inoue. Visual Navigation using Omnidirectional View Sequence. In Proc. of 1999 IEEE/RSJ Intl. Conf. on Intelligent Robots and Systems (IROS'99), pp. 317-322, 1999.

[5] H.Ishiguro, T.Miyashita, and S.Tsuji. T-Net for Navigating a Vision-Guided Robot in a Real World. In Proc. of IEEE Int. Conf. on Robotics and Automation, pp. 1068-1073, 1995.

[6] Y. Matsutmoto, T. Shibata, K. Sakai, M. Inaba, and H. Inoue. Real-time Color Stereo Vision System for a Mobile Robot based on Field Multiplexing. In Proc. of IEEE Int. Conf. on Robotics and Automation, pp. 1934-1939, 1997.

[7] H. Inoue, T. Tachikawa, and M. Inaba. Robot Vision System with a Correlation Chip for Real-time Tracking, Optical Flow and Depth Map Generation. In Proc. of the 1992 IEEE Int'l Conf. on Robotics and Automation, pp. 1621-1626, 51992. 